\title{
¿TIENEN LOS NIÑOS PENSAMIENTOS INTRUSOS ANÁLO- GOS A OBSESIONES? PRESENTACIÓN DE UN INSTRU- MENTO ESTANDARIZADO Y RESULTADOS PRELIMINARES
}

\author{
Lucía Igualada* \\ Conxa Perpiñáa* \\ Amparo Belloch"*
}

Servicio Valenciano de Salud, Departamento 12*

Facultad de Psicologia, Universidad de Valencla**

\section{RESUMEN}

Los pensamientos intrusos no deseados con contenidos similares a las obsesiones clínicas, constituyen una experiencia prácticamente universal en adultos sin psicopatologias. Sin embargo, no existen apenas evidencias de la presencia de estas intrusiones en población infantil y pre-adolescente. En este trabajo se presenta el Inventario de Pensamientos Intrusos Obsesivos para Niños y Adolescentes (INPIOS-NA), que recoge en 45 items la frecuencia con que se experimentan intrusiones con temática obsesivo-compulsiva. Lo completaron 122 nin̄os (12 y 13 años). El análisis factorial exploratorio produjo cuatro factores: 1) Agresión, sexo, acumulación; 2) Contaminación y dudas; 3) Superstición y Repetición; 4) Orden y Comprobación. La consistencia interna del instrumento fue excelente (valores de áe» 0,80). Los contenidos menos frecuentes fueron los del Factor 1. No hubo diferencias entre niños y niñas en la frecuencia con

Agradecimientos. Este estudio ha sido realizado gracias a la financiación recibida del Ministerio de Ciencia y Tecnologla y la aportación de fondos FEDER (Proyecto SEJ2006) 03893-PSIC).

Correspondencia: Prof Amparo Belloch. Departamento de Personalidad. Evaluación y Tratamientos Psicologicos. Facultad de Psicología. Avda. Blasco Ibález 21. Valencia-46010. E-mail: amparo.belloch@uv.es. 
que se experimentaban los distintos contenidos obsesivos (factores), pero los niños de 13 años informaron de más frecuencia de intrusiones de los factores 2,3 y 4 . El $90 \%$ de los sujetos dijo haber experimentado al menos alguna vez intrusiones obsesivas. Cuando se analizaron las tasas de frecuencia que entrarian en el rango de problemáticas, este porcentaje osciló entre el $8,2 \%$ (Factor 1) y el 15,57\% (Factor 3).

Palabras clave: PENSAMIENTOS INTRUSOS OBSESIVOS; TRASTORNO OBSESIVO-COMPULSIVO; OBSESIONES INFANTILES; COMPULSIONES INFANTILES.

\section{SUMMARY}

The unwanted intrusive thoughts with contents analogous to clinical obsessions are commonly experienced by non-clinical adults. However, there are not empirical evidences on the experience of these unwanted intrusions among children and preadolescents non clinical subjects. In this paper the ObsessionalIntrusive Inventory for Children and Adolescents is presented. It contains 45 items about obsessive-compulsive themes. Onehundred and twenty-two boys and girls, aged from 12 to 13 years, completed the questionnaire. The exploratory factorial analysis yielded 4 factors: 1) Aggression, sex, and hoarding; 2) Contamination and Doubts; 3) Superstition and Repetitive nituals; 4) Order and Checking. The intemal consistency was excellent ( a values e»0,80). The less frequently experienced contents were those included in Factor 1. There were not gender differences on the frequency with which the factors were experienced, but the children aged 13 years reported to have more intrusive thoughts from factors 2, 3, and 4. A 90\% of participants experienced at least one or two times the unwanted intrusions assessed. However, taking into account higher frequency rates (one or twice every month, or higher), the percentage of subjects who experienced the unwanted intrusions decreased (from $8.2 \%$ for the contents in factor 1 , to $15,57 \%$ for the contents in Factor 3 ).

Koy words: UNWANTED INTRUSIVE THOUGHTS; OBSESSIVE-COMPULSIVE DISORDER; CHILDREN'S OBSESSIONS; CHILDREN'S COMPULSIONS. 


\section{INTRODUCCIÓN}

La caracteristica esencial del Trastorno Obsesivo-Compulsivo (TOC) es la presencia recurrente y persistente de obsesiones, se acompañen o no de comportamientos compulsivos, tal y como se recoge en los criterios diagnósticos del DSM-IV (APA, 1994).

Aunque el TOC suele iniciarse en la adolescencia o a principios de la vida adulta, entre el $30-50 \%$ de pacientes adultos informan del inicio de sus síntomas durante la infancia (Rasmussen y Eisen, 1990). Las características clínicas del TOC en niños y adolescentes son similares a las del adulto, $y$ desde el punto de vista de los criterios diagnósticos, la diferencia fundamental estriba en que el adulto debe haber reconocido lo excesivo y/o irracional de sus obsesiones y/o compulsiones, al menos en algún momento de la evolución del trastomo. Sin embargo, este criterio no tiene porqué producirse en los niños. Desde el punto de vista clínico, esta diferencia es habitualmente apreciable, ya que en efecto la población infantil informa de una menor frecuencia en sus obsesiones, tal vez debido a su mayor dificultad en reconocer la naturaleza excesiva y/o irracional de las mismas. De hecho, suele ser habitual que sean los padres quienes informan de la presencia del problema, ya sea porque detectan en sus hijos un comportamiento extraño (rituales, comprobaciones excesivas, lentitud exagerada), del que, además, muy a menudo intentan hacer partícipes a los padres (Ulloa, de la Peña, Higuera, Palacios, Nicolini y Avila, 2004), ya sea porque encuentran extrañas y excesivas las preocupaciones que muestran sus hijos, y que se suelen traducir en preguntas u observaciones sobre temas poco comunes 0 habituales para la edad del niño, que además se reiteran de forma innecesaria o excesiva.

Las teorias actuales sobre el TOC en el ámbito cognitivocomportamental (i.e., Purdon y Clark, 1999; Rachman 1997, 1998; Salkovskis 1985,1989 ) plantean que los pensamientos obsesivos tienen sus ralces en algunos de los pensamientos cotidianos experimentados por personas «normales». Diversos estudios ponen de manifiesto que entre el $80-99 \%$ de las personas mentalmente sanas informan ocasionalmente de intrusiones que pueden ser experimentadas como pensamientos, imágenes o impulsos (Clark y 
de Silva, 1985; Freeston, Ladouceur, Gagnon y Thibodeau, 1991; Freeston, Ladouceur, Thibodeau y Gagnon, 1992; Niler y Beck, 1989; Parkinson y Rachman, 1981; Purdon y Clark, 1993, 1994a,b; Reynolds y Salkovskis, 1991; Salkovskis y Harrison, 1984). Más recientemente, también en nuestro contexto (Belloch, Morillo, Lucero, Cabedo, $Y$ Carrió, 2004; Lucero, 2002) se ha podido constatar que la mayoria de las personas pertenecientes a la población adulta sana reconocian haber experimentado alguna vez un pensamiento intruso, con contenido análogo a las obsesiones clínicas. Este tipo de productos cognitivos, denominados habitualmente pensamientos intrusos obsesivos (PIOs), se consideran los análogos normales de las obsesiones clínicas, y su análisis está permitiendo profundizar en el conocimiento y abordaje de la psicopatología obsesivo-compulsiva (Clark, 2005)

Rachman (1981) propuso una definición ampliamente aceptada de este tipo de pensamientos: "pensamientos, imágenes o impulsos repetitivos que no son deseados ni aceptados y que a) interrumpen la actividad en curso, b) son internamente atribuidos; y c) son dificiles de controlar». Por otra parte, Clark y Purdon (1995; Purdon y Clark, 1999) señalaron que algunos de los instrumentos empleados en los estudios sobre este tipo de pensamientos en la población general tienen problemas de validez de constructo, ya que están basados en definiciones demasiado globales de tales pensamientos, confundiéndose a veces con contenidos depresivos (automáticos) o ansiosos (preocupación patológica o "worry»). En este sentido, Clark y $\mathrm{O}^{\prime}$ Connor (2005), utilizando medidas de entrevista individualizada para la detección de PIOs, constataron que únicamente el $11 \%$ de los entrevistados (población no clínica) referían de manera espontánea experimentar este tipo de intrusiones, frente a una inmensa mayoría que relataba intrusiones de tipo ansioso no obsesivo (preocupaciones tipo "worty", principalmente). Algunos estudios empiricos recientes han postulado que la dimensión egodistonia-egosintonía en relación al contenido del pensamiento intruso, constituye de hecho una característica fundamental para diferenciar entre PIOs y pensamientos más típicos de preocupaciones de naturaleza ansiosa en general (Langlois, Freeston, y Ladouceur, 2000a,b; Turner, Beidel y Stanley, 1992).

Asi pues, resulta especialmente importante disponer de instrumentos de evaluación que permitan verdaderamente apresar 
y valorar la presencia de auténticos pensamientos intrusos análogos a las obsesiones clínicas. Purdon y Clark (1993, 1994 a,b) diseñaron el Revised Obsessional Intrusions Inventory (ROII), que ha demostrado ser un instrumento autoaplicado útil y válido para diferenciar de manera adecuada entre PIOs y otros tipos de pensamientos intrusos no obsesivos (por ejemplo, Pensamientos automáticos negativos, característicos de la depresión, o pensamientos sobre preocupaciones cotidianas de tipo "worry", caracteristicos de la ansiedad generalizada). Este instrumento ha sido adaptado y validado para su uso en población española adulta, y ha demostrado su utilidad tanto en población general no clínica, como en población clínica, es decir, ha permitido distinguir entre pacientes TOC, depresivos, y personas con otros trastornos de ansiedad diferentes al TOC (Belloch et al., 2004; Giménez, Morillo, Belloch, Lucero, Carrió y Cabedo, 2003; Morillo, Belloch y Garcia-Soriano, 2006; Morillo, Giménez, Belloch, Lucero, Carrio, y Cabedo, 2003). Sin embargo, no existe ningún instrumento para la valoración de este tipo de intrusiones en la población infantil.

Teniendo en cuenta lo expuesto, el objetivo fundamental de este trabajo ha sido diseñar un instrumento que permitiera detectar la presencia de Pensamientos Intrusos análogos a Obsesiones (PIOs) en población infantil (edad mínima 12 anos), de tal forma que se puedan diseñar posteriormente estudios de mayor alcance para evaluar la frecuencia real de estos pensamientos, sus contenidos más habituales, así como las características de los más egodistónicos (contenido, frecuencia, grado de malestar).

\section{MÉTODO}

\section{Participantes}

Han participado 120 escolares procedentes de la población general, escolarizados en dos centros públicos de la provincia de Valencia, de los que 64 eran niños $(53,3 \%)$ y 56 niñas $(46,7 \%)$. El $45 \%$ de la muestra (29 niños y 25 niñas) cursaba $1^{\circ}$ de la E.S.O., y la edad media (DT) era de $12,3(0,5)$ años. El restante $55 \%$ ( 35 niños y 31 niñas) estaba en $2^{\circ}$ de la E.S.O. y tenía una edad media de $13,43(0,72)$ años. 
El $71,7 \%$ de los niños indicó tener al menos otro hermano (rango entre 1 y 8 hermanos), ocupando el primer lugar en la fratria en un $45 \%$ de los casos. El $13,3 \%$ era hijo único. El $76,7 \%$ de las familias presentaba un nivel socioeconómico medio. Por último, la nacionalidad de los padres era predominantemente española.

\section{Instrumentos}

Inventario de Pensamientos Intrusivos Obsesivos para Niños y Pre-Adolescentes (INPIOS-NA)

Partiendo de instrumentos tales como el Inventario Obsesivo Compulsivo Revisado II el (The Revised Obsessive Intrusions Inventory, ROIl; Purdon y Clark, 1994) y la Escala de Obsesiones y Compulsiones para niños y adolescentes de Yale-Brown (Children's Yale-Brown Obsessive Compulsive Scale, CY-BOCS, Scahill, Riddle, Hardin, Ort, Goodman, Price, et al, 1997), se procedió a diseñar y adaptar items para la población infantil y adolescente. Se pidió colaboración en esta elaboración a varios especialistas en psicopatología y en la clínica del TOC, pertenecientes al grupo de investigación en el que se enmarca este trabajo. Por último, se pusieron en común los listados de ítems y se consensuaron los que deberían incluirse en el inventario. Se utilizó como formato de respuesta una escala tipo Likert de 7 puntos entre 0 (Nunca: «Nunca he tenido ese pensamiento o comportamiento antipático») y 6 (Siempre: «Pienso en esto continuamente, durante todo el día, o muchas veces al dia»). Finalmente, el cuestionario asi elaborado se compuso de 43 items agrupados según situaciones desencadenantes (escenarios). Además habia dos ítems idiosincrásicos (44 y 45) que se contestaban si procedía, y con el mismo formato de respuesta. Por último, se pedia a los niños que escogiesen el pensamiento intruso que le resultaba más desagradable o molesto de entre todos los valorados con una puntuación superior a cero.

\section{Procedimiento}

Se explicó el objetivo del estudio con mayor profundidad a los psicólogos y profesores de aquellos colegios que quisieron participar de forma voluntaria. Se pidió la colaboración de los tutores para repartir 
la hoja informativa y el consentimiento entre los alumnos, quienes, para formar parte del estudio, debían traer el consentimiento informado de los padres, además de una pequeña encuesta contestada por ellos en los que se verificaba que los niños participantes no informaran de trastomo mental diagnosticado.

El proceso de recogida de datos y evaluación se hizo, en todos los casos, de manera colectiva en pequeños grupos y en horas de clase, en presencia de una de las autoras (L.I.), quien además revisaba que todos los participantes completaran adecuadamente el cuestionario y respondia a las dudas que pudieran tener.

\section{RESULTADOS}

\section{Estructura factorial y flabilidad del INPIOS-NyA}

Con el fin de analizar la composición del nuevo inventario se realizó un análisis factorial exploratorio de componentes principales (rotación Varimax). Se utilizaron los criterios siguientes para la composición y retención de factores: valor propio d" 1, carga factorial (saturación)e» 0,30 para la inclusión de un item en el factor, contenido interpretable de los factores, y un mínimo de 5 items por factor. Los items 44 y 45 , de contenido idiosincrásico, no se incluyeron en el análisis dado que solo 15 participantes hablan registrado intrusiones particulares.

Según estos criterios, la mejor estructura factorial fue la que agrupó todos los items en cuatro factores que, en conjunto, explicaron el $44,14 \%$ de la variarıa total, que se distribuyó de manera bastante homogénea en los 4 factores obtenidos. El primer factor incluyó 13 items referidos a intrusiones sobre comportamientos auto- o heteroagresivos, sexuales, comportamientos socialmente inadecuados, y acumulación. El segundo factor, también con 13 items, agrupó intrusiones sobre contaminación y dudas. El factor 3 recogió 9 items referidos a supersticiones-pensamiento mágico, y rituales de comprobación. Y el cuarto y último factor con 8 items sobre intrusiones de orden y comprobación. No obstante, cabe señalar que seis items $(1,2,23,24,35$ y 41$)$ presentaron saturaciones dentro del nivel exigido en dos factores. La inclusión en uno de ellos se decidió sobre la base del contenido del item. 
La fiabilidad (consistencia interna) de la puntuación total del cuestionario fue excelente (? de Cronbach $=0,92$ ). Asimismo, la consistencia interna de cada uno de los cuatro factores arrojo valores entre buenos y excelentes, y no fue necesaria la eliminación de ningún item teniendo en cuenta la aportación al valor final de á. En la Tabla 1 se resumen estos resultados, junto con una descripción breve de cada uno de los items para facilitar la comprensión.

\section{Tabla 1. Estructura factorial del INPIOS-NA}

\begin{tabular}{|c|c|c|}
\hline Item & $\begin{array}{l}\text { FACTOR 1: Agresión-Sexo-Acumulación } \\
\text { (Vorianza explicada }=11,70 \% ; \alpha=0,82)\end{array}$ & Saturación \\
\hline$\overline{4}$ & Hacerme dano a propósito & 0,33 \\
\hline 5 & Hacer daño a alguien a propósito & 0,51 \\
\hline 6 & Tirarme a los coches, al metro, o al autobús & 0,50 \\
\hline 7 & Empujar a alguien desconocido a los coches, o al metro... & 0,53 \\
\hline 8 & Sin que me provoquen, dar un golpe, una patada, un tortazo... & 0,53 \\
\hline 9 & Sin que me provoquen, insultar, dar un grito para que se asusten & 0,55 \\
\hline 10 & Sin que me provoquen, robar, aunque no me guste ni lo necesite & 0,68 \\
\hline 11 & Romper o estropear algo a propósito & 0,62 \\
\hline 12 & $\begin{array}{l}\text { Pensamientos, imágenes o impulsos sexuales asquerosos, malos, dan } \\
\text { vergüenza }\end{array}$ & 0,62 \\
\hline 13 & $\begin{array}{l}\text { Decir en voz alta una cochinada, un insulto, una burrada, una } \\
\text { blasfemia, etc }\end{array}$ & 0,64 \\
\hline 34 & Guardar cosas aunque ya no me sirvan, no me gusten, o estén viejas & 0,40 \\
\hline 42 & Hacer daño adrede a alguien de mi familia o a un amigo & 0,63 \\
\hline 43 & Insultar o provocar a alguien de mi familia o de mis amigos & 0,60 \\
\hline \multicolumn{3}{|c|}{$\begin{array}{l}\text { FACTOR 2: Contaminacion-Dudas } \\
\text { (Varianza explicada }=11,35 \%, a=0,85) \text { ) }\end{array}$} \\
\hline 1 & Estar sucio o contaminado aunque no haya tocado nada sucio & 0,41 \\
\hline 2 & $\begin{array}{l}\text { Que me he podido contaminar, o enfermedad, al tocar planta, bolsa } \\
\text { basura, peine... }\end{array}$ & 0,40 \\
\hline 3 & $\begin{array}{l}\text { Que me he podido contaminar, o ...enfermedad, al tocar algo que han } \\
\text { tocado desconocidos }\end{array}$ & 0,46 \\
\hline 14 & Que haya un incendio en mi casa, que entren a robar & 0,52 \\
\hline 15 & Que sin darme cuenta, contagie a alguien de una enfermedad & 0,57 \\
\hline 16 & Que se me pierda algo que me gusta mucho, o que vale mucho & 0,51 \\
\hline 17 & $\begin{array}{l}\text { Que le ocurra algo malo a alguien de mi familia porque no tuve } \\
\text { cuidado }\end{array}$ & 0,74 \\
\hline 19 & Si habré hecho bien algo & 0,57 \\
\hline 20 & Si habré hecho algo mal y eso puede causar una desgracia & 0,60 \\
\hline 24 & Si yo habré tenido algo que ver en esa desgracia & 0,54 \\
\hline 25 & Si yo podia haber hecho algo para evitar que pasara... & 0,58 \\
\hline 28 & Revisar de nuevo la cerradura de la cartera, de la casa, etc... & 0,67 \\
\hline 31 & Asegurarme de que mis cosas están en el orden que quiero & 0,51 \\
\hline
\end{tabular}


Tabla 1. Estructura factorial del INPIOS-NA (continuación)

\begin{tabular}{|c|c|c|}
\hline Item & $\begin{array}{l}\text { FACTOR 3: Superstición-Repeticion } \\
\text { (Varianza explicada }=10,76 \% ; a=0,85)\end{array}$ & Saturación \\
\hline 18 & $\begin{array}{l}\text { Que si no toco o hago algo ..veces seguidas_(o si no pienso algo), } \\
\text { puede pasar desgracia }\end{array}$ & 0,66 \\
\hline 21 & $\begin{array}{l}\text { Que pueden pasar cosas malas si he visto un gato negro, dos } \\
\text { coches de un color... }\end{array}$ & 0,65 \\
\hline 22 & $\begin{array}{l}\text { Que puedan pasar...cosas malas, si hago ciertas cosas ..., o si dejo } \\
\text { de hacer otras }\end{array}$ & 0,71 \\
\hline 32 & Repetir algunas cosas & 0,60 \\
\hline 33 & Contar varias veces y en un orden determinado & 0,60 \\
\hline 35 & Tocar varias veces un objeto, o a una persona, o a ml mismo & 0,55 \\
\hline 36 & Evitar ciertas cosas porque pueden traer mala suerte o desgracias & 0,80 \\
\hline 37 & Abrir o cerrar las puertas con los codos & 0,47 \\
\hline \multirow[t]{2}{*}{41} & $\begin{array}{l}\text { Hacer cosas mentalmente (contar, rezar, cantar ...), antes de hacer } \\
\text { algo ... }\end{array}$ & 0,34 \\
\hline & $\begin{array}{l}\text { FACTOR 4: Orden-Comprobación } \\
\text { (Varianza explicada }=10,34 \% ; a=0,80)\end{array}$ & \\
\hline 23 & $\begin{array}{l}\text { Que ciertas cosas.. no correctamente ordenadas, no guardan un } \\
\text { orden concreto ... }\end{array}$ & 0,36 \\
\hline 26 & $\begin{array}{l}\text { Aunque sé que está limpio...lavarme varias veces seguidas las } \\
\text { manos }\end{array}$ & 0,59 \\
\hline 27 & $\begin{array}{l}\text { Aunque sé que está limpio...limpiar otra vez mi ropa, mis libros, la } \\
\text { mochila... }\end{array}$ & 0,62 \\
\hline 29 & Comprobar varias veces que llevo libros, cuademos... & 0,38 \\
\hline 30 & $\begin{array}{l}\text { Aunque sé que no es necesario...comprobar varias veces lo que he } \\
\text { escrito }\end{array}$ & 0,40 \\
\hline 38 & $\begin{array}{l}\text { Cepillarme ... un determinado número de veces, y siguiendo un } \\
\text { orden concreto }\end{array}$ & 0,66 \\
\hline 39 & $\begin{array}{l}\text { Botar la pelota un número ...de veces antes de pasarla, ...no me } \\
\text { saldrá bien el tiro }\end{array}$ & 0,60 \\
\hline 40 & Preguntar lo mismo varias veces seguidas ... & 0,40 \\
\hline
\end{tabular}

\section{Frecuencia real con la que se experimentan PIOS}

El procedimiento habitual para calcular los estadísticos descriptivos de un cuestionario en el que se mide la frecuencia temporal con la que aparece y/o se experimenta lo que se pretende valorar, es tomar en consideración la suma directa de las puntuaciones individuales. No obstante, este procedimiento tiene el inconveniente de no tener en cuenta en qué medida los sujetos realmente experimentan aquello que se les pregunta, sobre la base de la escala de respuesta que se les 
propone. Con el fin de paliar este problema, que proporciona un panorama equivocado de la realidad que se pretende analizar, calculamos la frecuencia real con la que se experimentaban los pensamientos intrusos propuestos, según la fórmula siguiente: $\mathrm{N}^{\circ}$ de pensamientos que se experimentan con alguna frecuencia (puntuaciones e»1, "una o dos veces en toda mi vida») / suma directa de las puntuaciones registradas para ese pensamiento. Es decir, se eliminan del cómputo los casos en los que la respuesta es 0 («nunca»), y el dato final resultante indica la situación del conjunto de items o subescala en la escala de frecuencia real (en nuestro caso, fluctúa desde $1=$ «una $\circ$ dos veces en toda mi vida», hasta $6=$ "muchas veces, todos los días"). Además, la fórmula permite saber con precisión el número de participantes que realmente experimentan, aunque solo sea alguna vez, pensamientos intrusos análogos a obsesiones. En la Tabla 2 se resumen los estadisticos obtenidos a partir de este procedimiento, asI como la media no ponderada (suma directa de las puntuaciones) para cada uno de los cuatro factores extraldos.

Como puede observarse en la Tabla, más del $90 \%$ de los participantes expresaron tener al menos alguna vez los pensamientos intrusos evaluados. El mayor porcentaje de niños que decia experimentar estos pensamientos recayó en el factor 2 (Contaminación y Dudas), mientras que el menor número se centró en el factor 3 (Superstición y Repetición). Por lo que se refiere a la frecuencia con la que se experimentaban los pensamientos propuestos, la menor puntuación recayó en el primer factor (agresión sexo, conductas sociales inadecuadas, acumulación), ya que los participantes relataron experimentar este tipo de pensamientos entre casi nunca («una o dos veces en toda mi vida») y alguna vez ( "alguna vez al año»). Sin embargo, el resto de intrusiones eran más frecuentes, aunque como es lógico, relativamente raras. No obstante, se observaron frecuencias máximas muy elevadas en todos los factores $(5=$ «lo pienso todos los dias alguna vez"), lo que indica que una proporción pequeña de sujetos experimentaba PIOs con mucha mayor frecuencia de lo esperado, al tratarse de una población escolar normal.

Por otra parte, si se tiene en cuenta que puntuaciones superiores a 3 indican una frecuencia de aparición de intrusiones relativamente alta («una o dos veces al mes»), el número real de niños que obtuvo 
Tabla 2. Estadísticos descriptivos del INPIOS-NA

\begin{tabular}{lcccr}
\hline FACTORES (sujedos) & $\begin{array}{c}\text { Media } \\
(D) \\
(N=122)^{a}\end{array}$ & $\begin{array}{c}\text { Frecuencia } \\
\text { Media }(D)\end{array}$ & $\begin{array}{c}\text { Percentiles } \\
75-85-90\end{array}$ & $\begin{array}{c}\text { Frecuencia } \\
\text { máxima }\end{array}$ \\
\hline 1. Agresión, sexo, acumulación & $8,8(7,80)$ & $1,86(0,76)$ & $2,33-2,53-3,29$ & 5 \\
$(\mathrm{~N}=111)$ & & & & \\
2. Contaminación, Dudas, $(\mathrm{N}=117)$ & $14,6(9,66)$ & $2,02(0,79)$ & $2,32-2,86-3,25$ & 4,6 \\
3. Superstición, Repetición $(\mathrm{N}=102)$ & $8,26(8,6)$ & $2,02(1,01)$ & $2,5-3,18-3,46$ & 5,4 \\
4. Orden, Comprobación $(\mathrm{N}=115)$ & $10(7,6)$ & $2(0,88)$ & $2,37-3-3,41$ & 5 \\
\hline
\end{tabular}

${ }^{a}$ En este caso el $N$ corresponde a la totalidad de la muestra.

- Percentiles calculados sobre la frecuencia media real

puntuaciones elevadas en cada uno de los factores fue el siguiente: 10 niños en el Factor 1 ( $8,2 \%$; percentil 90$)$, 18 en el Factor $2(14,75 \%$; percentil 90), 19 en el Factor 3 (15,57\%; percentil 85$)$, y de nuevo 18 niños en el Factor 4 (14,75\%; percentil 85$)$. Estos resultados indican que en una proporción pequeña pero significativa de niños, la presencia de pensamientos intrusos análogos a obsesiones clínicas se produce con una frecuencia excesiva, lo que indicaria la necesidad de una exploración psicológica más detenida para valorar la presencia de un trastorno obsesivo-compulsivo de inicio temprano.

\section{Diferencias entre niños y niñas y entre niveles educativos}

En primer lugar se comprobó que no existian diferencias sociodemográficas entre $1^{\circ}$ y $2^{\circ}$ de la E.S.O., ni entre chicos y chicas, en edad, número de hermanos, porcentaje de género en ambos cursos, nivel socioeconómico y nivel de estudios de los padres.

Con el fin de examinar si existían diferencias entre sexos y entre grupos de edad (cursos), se calcularon pruebas $t$ (muestras independientes) tomando como referencia la frecuencia media real en cada uno de los factores y en la puntuación total. No se constataron diferencias entre la frecuencia real con la que los niños y las nifias experimentaban los pensamientos intrusos, por lo que, al menos en estos niveles de edad, no se aprecian diferencias de género.

Sin embargo, los niños más mayores, es decir, los que cursaban $2^{\circ}$ de ESO, obtuvieron puntuaciones significativamente más elevadas 
en todos los los factores a excepción del primero (Agresión, sexo, acumulación), en el que las diferencias no fueron significativas. Estos datos puedes estar indicando que a partir de los 13 años comienzan a experimentarse con una cierta frecuencia intrusiones con contenidos típicamente obsesivos, 0 , alternativamente, que es a esa edad cuando los niños son capaces de darse cuenta de que están experimentando pensamientos, impulsos o imágenes no deseados.

Tabla 3. Diferencias entre cursos escolares en los factores del INPIOS-NA

\begin{tabular}{lllll}
\hline Factor & $\begin{array}{l}\text { Curso } \\
\text { escolar }\end{array}$ & Media (DT) & $t$ & $p$ \\
& & & \\
\hline 1. Agresión, Sexo, Acumulación & $1^{\circ} \mathrm{ESO}$ & $1,81(0,75)$ & & \\
& $2^{\circ} \mathrm{ESO}$ & $1,89(0,76)$ & $-0,61$ & 0,54 \\
& $1^{\circ} \mathrm{ESO}$ & $1,85(0,85)$ & & \\
2. Contaminación-Dudas & $2^{\circ} \mathrm{ESO}$ & $2,15(0,72)$ & 2,01 & 0,04 \\
& $1^{\circ} \mathrm{ESO}$ & $1,77(0,84)$ & & \\
3. Superstición-Repetición & $2^{\circ} \mathrm{ESO}$ & $2,22(1,10)$ & 2,30 & 0,02 \\
& $1^{\circ} \mathrm{ESO}$ & $1,78(0,76)$ & & \\
4. Orden-Comprobación & $2^{\circ} \mathrm{ESO}$ & $2,17(0,94)$ & 2,34 & 0,02 \\
\hline
\end{tabular}

Contenidos de PIOs más frecuentes en población escolar

Con el fin de examinar cuáles eran los pensamientos intrusos que los niños experimentaban con mayor frecuencia, se seleccionaron los items del INPIOS-NA que registraron las mayores frecuencias reales. La selección se hizo de acuerdo con dos criterios, del mismo modo que ya se hizo en un trabajo anterior con población adulta normal (Belloch et al., 2004): a) que estuvieran entre los ítems con mayor puntuación media, y b) los que acumularan mayor porcentaje en las categorias de respuesta 4: «A menudo» (una o dos veces por semana), 5: «Muy a menudo (a diario) y 6: "Siempre»(frecuentemente durante todo el día). En la Tabla 4 se muestran los 10 ítems más comunes 0 frecuentes. 
Tabla 4. Contenidos de PIOs que se experimentan con más frecuencia

\begin{tabular}{lcc}
\hline $\mathrm{N}^{\circ}$ ítem - Pensamiento Intruso & Media (DT) & $\%{ }^{(\mathrm{a})}$ \\
\hline 16- Que se me pierda algo que me gusta mucho... & $2,06(1,64)$ & 20 \\
34- Guardar cosas aunque ya no sirvan, ... & $1,93(1,81)$ & 20,1 \\
14- Que haya un incendio en mi casa, o que entren a robar,.. & $1,83(1,48)$ & 15,8 \\
41- Hacer algunas cosas mentalmente... antes de hacer algo & $1,71(1,77)$ & 15,8 \\
28- Revisar de nuevo la cerradura, el interruptor, el grifo... & $1,88(1,59)$ & 14,2 \\
*29- Conprobar varias veces que llevo libros, cuadernos, etc., & $1,78(1,56)$ & 14,2 \\
17- Que le ocurra algo malo a alguien de mi familia porque no tuve cuidado & $1,73(1,54)$ & 12,5 \\
32- Repetir algunas cosas .. & $1,18(1,53)$ & 10,8 \\
*23- Que ciertas cosas no correctamente ordenadas, ..no orden concreto & $1,05(1,53)$ & 10,1 \\
*30- Aunque se que no es necesario...comprobar varias veces lo escrito & $1,63(1,33)$ & 9,2 \\
\hline
\end{tabular}

(a) Porcentaje de niños que responden al item entre 4 y 6 (El pensamiento se da con una frecuencia entre "a menudo» y «siempre»)

$\left.{ }^{\star}{ }^{\star}\right)$ Los items marcados con un asterisco fueron más frecuentes en niños que en niñas.

Como puede observarse, la mayor parte de estos contenidos más frecuentes hacen referencia a dudas, orden, y comprobación. No obstante, resulta llamativa la frecuencia con las que se experimentaba el item 34 , que hace referencia a acumulación de objetos inútiles.

\section{Análisis del PIO Más Molesto: Contenidos y Frecuencia}

En las instrucciones del instrumento, una vez que el niño había respondido al listado inicial de PIOs, se le pedía que escogiera el PIO de la lista (contando con los idiosincrásicos, si los hubiera), que más molesto le resultara cuando lo tenia.

Hubo amplias diferencias individuales a la hora de seleccionar el PIO más molesto. Las niñas seleccionaron 21 pensamientos diferentes, y los niños 31. Las niñas escogieron más PIOs del Factor 2 (Dudas y Contaminación) que los niños. Sin embargo, los niños escogieron sobre todo pensamientos incluidos en el factor 1 (Agresión, Sexo, Acumulación, Conductas inadecuadas). Además, 8 niñas y 7 niños eligieron como PIO 
más molesto los idiosincrásicos, es decir, aquellos que experimentaban pero que no se hallaban recogidos en el listado del cuestionario.

Entre estos contenidos idiosincrásicos, resulta destacable que cinco de ellos, es decir, la mayoria, hiciera referencia a temas relacionados con la muerte («caer al vacío desde un balcón» «que personas queridas pueden morir» " pensar en el día de mi muerte» «imagen de verme enfermo o muerto" "pensamientos de suicidio»). Otros 3 hacian referencia a dudas/comprobación especificas («si me han llamado al móvil» «me he dejado las llaves o el móvil en algún sitio» "he olvidado algo»), y otro se podría clasificar en el grupo de superstición y pensamiento mágico («puede ocurrirme lo que sale en las noticias, como por ejemplo que muera»). El resto de pensamientos particulares o idiosincrásicos no eran clasificables como análogos obsesivos, sino como pensamientos automáticos negativos («no tengo ninguna cualidad»), preocupaciones de tipo «worry» («a algún familiar o amigo le puede pasar algo malo que he visto o escuchado en la tele», "a alguien querido le va a pasar algo malo»), y por último, confusión entre lo que se preguntaba (PIOs) y miedos, inseguridades, o temores («que otros me mienten» «que me puede ocurrir otra vez algo que ya me ha pasado» "sobre películas de miedo»).

En conjunto, estos resultados indican que los niños entendieron el propósito del cuestionario correctamente, puesto que la mayoría de estos «pensamientos idiosincrásicos» hacian referencia efectivamente a contenidos obsesivos, y solo en tres casos los contenidos eran clasificables más como intrusiones de tipo depresivo o características de ansiedad generalizada ( worry»).

Por otro lado, observamos que en el $24,16 \%$ de los casos el PIO escogido como más molesto se experimentaba con una frecuencia muy elevada, ya que en la escala de respuesta se calificaba entre 5 ("lo pienso todos los días alguna vez») y 6 ("pienso en esto continuamente, durante todo el dia, o muchas veces al dia»).

\section{Análisis de los PIOs que no suelen experimentarse.}

Dado que se está analizando la presencia de este tipo de pensamientos en población «general» escolar, se procedió a explorar qué tipo de PIOs no son, o no suelen ser, experimentados por estos 
niños. Para ello se escogieron aquellos pensamientos que fueron catalogados con respuestas 0: «Nunca he tenido este pensamiento 0 comportamiento» por la mayoría de los niños (75\% o más). En total fueron 7 items (números $5,6,7,10,15,37$ y 42) los que, tanto los niños como las niñas, dijeron no haber tenido nunca. Cinco de estos pensamientos hacen referencia a comportamientos violentos y agresivos contra los demás o contra uno mismo, y los otros dos hacen referencia a miedos a trasmitir/contraer una enfermedad.

\section{DISCuSIóN}

El objetivo principal de este trabajo ha sido explorar la presencia de pensamientos intrusos no deseados análogos a obsesiones en población infantil y pre-adolescente, ya que la totalidad de estudios publicados al respecto desde el trabajo pionero de Rachman y de Silva (1978) se ha centrado exclusivamente en poblaciones adultas normales. Para ello, se ha diseñado un cuestionario, que hemos denominado Inventario de Pensamientos Intrusos Obsesivos en riños y adolescentes (INPIOS-NA), dada la ausencia de instrumentos estandarizados que permitieran explorar nuestro objetivo.

Los resultados psicométricos iniciales que se presentan en este estudio indican que el nuevo cuestionario es útil y válido para explorar la presencia de PIOs en niños sin historia de problemas mentales a partir de 12 años. Además, los cuatro factores en que se agrupan los items del cuestionario hacen referencia clara a subtipos de contenidos obsesivos, iguales a los que se encuentran en población adulta. Las propiedades psicométricas exploradas indican que el cuestionario es fiable, lo que garantiza en buena medida la viabilidad de los resultados. Por otro lado, el cuestionario fue bien comprendido por los niños, lo que indica que las instrucciones eran correctas y que ya en estas edades es posible encontrar intrusiones análogas a las obsesiones y compulsiones clínicas. De hecho en torno al $90 \%$ de los encuestados reconoció haber experimentado alguno o más de los PIOs en más de una ocasión, en especial cuando se trata de contenidos relacionados con Contaminación, Dudas, Superstición, Repetición, Orden, y Comprobación. Mucho menos frecuentes fueron los relacionados con contenidos agresivos, sexuales, o socialmente inadecuados. 
No se detectaron diferencias entre niños y niñas en cuanto a la frecuencia real con la que se experimentaban los cuatro tipos (factores) de PIOs. Este resultado es distinto al detectado en población adulta general, ya que se ha constatado que los hombres suelen presentar unas tasas de pensamientos intrusos obsesivos superior a la de las mujeres, en especial los referidos a temas de agresión, sexo, y comportamientos socialmente inadecuados (Belloch et al., 2004; Purdon y Clark, 1993, 1994a). En este trabajo, estos contenidos, que se agrupan en el Factor 1, fueron de hecho los que presentaron una menor frecuencia real de aparición, lo que indica que quizá la ausencia de diferencias entre niños y niñas en estos contenidos, guarda relación con la edad. No obstante, a la hora de seleccionar el PIO que resultaba más molesto, las niñas tendleron a escoger intrusiones del Factor 2 (Contaminación, Dudas), mientras que los niños escogieron más PIOs del Factor 1 (Agresión, sexo, acumulación, y conductas inadecuadas socialmente). En este nivel de análisis, por tanto, los resultados sí que resultan equiparables a los detectados en adultos.

En todo caso, lo que sí que observamos con claridad fue la existencia de claras diferencias entre $1^{\circ}$ y $2^{\circ}$ de la E.S.O. en todos los factores, a excepción del primero, siendo en todos los casos los niños más mayores quienes obtuvieron una puntuación media más elevada en comparación con los más pequeños. Teniendo en cuenta que la media de edad de los niños que formaban el grupo de $2^{\circ}$ de la ESO era de 13 años, lo que se corresponde a la pre-adolescencia, es comprensible que sea en esta edad donde comienzan a aparecer dudas e inseguridades acerca de los propios comportamientos y/o actitudes. Es bien sabido que la pre-adolescencia constituye un periodo del desarrollo en buena medida "caótico", durante el cual se lucha por desarrollar una identidad propia, especialmente frente a los pares, a la vez que se producen los primeros reclamos hacia los familiares de un espacio vital propio e independiente, sin obviar por supuesto las necesidades de adaptación a los cambios de todo tipo que se producen en su cuerpo. Además, es seguramente en esta época en donde se comienza a ser verdaderamente consciente de las propias operaciones y productos mentales, entre los cuales los pensamientos ocupan como es natural un lugar preponderante. Este estudio no fue diseñado para poner a prueba este tipo de planteamientos. No obstante, 
dados los resultados obtenidos, y con todas las precauciones propias de una investigación como la presente, cabe pensar que la edad de los 13 años constituye un momento clave para la experiencia consciente de pensamientos intrusos análogos a las obsesiones clínicas.

Los contenidos de las intrusiones más frecuentes fueron similares a los encontrados en población adulta (Belloch et al., 2004; Purdon y Clark, 1993, 1994a), y hacian referencia a dudas, comprobaciones, necesidad de repetir y de ordenar. No obstante, resulta llamativo el hecho de que el ítem que recogía tendencias de acumulación resultara también muy frecuente, sobre todo porque el acumulador es uno de los subtipos del TOC con mayor sinificado patológico (Frost y Hartl, 1996). En nuestra opinión, en el caso de los niños sería excesivo otorgarle un significado excesivamente patológico, dado que en estas edades las necesidades de guardar objetos aunque se sepa que ya son inservibles, es un comportamiento muy habitual.

Mención aparte merece el hecho de que entre las intrusiones idisosincrásicas, no recogidas por tanto en el cuestionario, ocupen un lugar destacado las referidas a la muerte. Como señalaron Toro, Cervera, Osejo y Salamero (1992), estos contenidos son muy habituales en la población escolar, y su incidencia puede guardar relación con el hecho de que a estas edades, a diferencia de las inmediatamente anteriores, se ha alcanzado una comprensión plena de lo que significa la muerte, por lo que puede que su saliencia entre las preocupaciones obsesivas de los niños se deba a esta comprensión. En todo caso, nos parece importante incluir items con estos contenidos en el INPIOS-NA para futuros estudios.

Por lo que se refiere a los PIOs no experimentados, cabe resaltar que cinco de los 7 contenidos de estos pensamientos hacian referencia a comportamientos violentos y agresivos contra los demás o contra uno mismo, y los otros dos a miedos a trasmitir y/o contraer una enfermedad.

Finalmente, por lo que se refiere a las limitaciones de este trabajo, debemos destacar en primer lugar el hecho de que se haya explorado la presencia de intrusiones análogas a obsesiones mediante un cuestionario estandarizado. Si bien es cierto que los cuestionarios autoaplicados son relativamente económicos, consumen menos 
tiempo y pueden aplicarse a grupos grandes de personas, existe mayor dificultad en cuanto a la definición e interpretación de los conceptos, por lo que hay que tener cautela en pretender generalizar los resultados. Además, las respuestas tienen carácter retrospectivo, lo que supone una mayor susceptibilidad a sesgos como los de memoria, deseabilidad social, o de empleo de determinados patrones de respuesta. Una segunda limitación, quizá más importante, también relacionada con el uso de cuestionarios autoaplicados, es el hecho de que se requiere cierto nivel de capacidad introspectiva para darse cuenta de lo que se está preguntando: pensamientos intrusos no deseados. $Y$ es posible que algunos niños no hayan adquirido todavía esa capacidad, por lo que sus respuestas pueden ser erráticas 0 impredecibles. Por otro lado, el tamaño reducido de la muestra, no permite extraer conclusiones de mayor alcance desde el punto de vista de la generalización de los resultados.

A pesar de las limitaciones apuntadas, pensamos que el INPIOSNA viene a rellenar una laguna importante en torno a la investigación del TOC, y los resultados obtenidos, aun siendo preliminares, son los bastante prometedores como para diseñar estudios de mayor alcance que permitan avanzar no solo en la fiabilidad y la validez del cuestionario, sino además en el conocimiento del TOC en etapas tempranas de la vida.

\section{REFERENCIAS BIBLIOGRÁFICAS}

American Psychiatric Association (1994). Manual Diagnóstico y Estadístico de los Trastornos Mentales. Barcelona: Masson Belloch, A., Morillo, C., Lucero, M., Cabedo, E. Y Carrió, C. (2004). Intrusive thoughts in non-clinical subjects: the role of frequency and unpleasantness on appraisal ratings and control strategies. Clinical Psychology and Psychotherapy 11, 100-110.

Clark, D.A. (2005). Intrusive thoughts in clinical disorders. Theory, Research and Treatment. New York: Guilford Press.

Clark, D.A., y de Silva, P. (1985). The nature of depressive and anxious thoughts: Distinct or uniform phenomena?. Behaviour Research and Therapy, 23, 383-393.

Clark , D.A. y O'Connor, K. (2005). Thinking is believing: Ego-dystonic 
intrusive thoughts in obsessive-compulsive disorders. In D.A. Clark (ed.). Intrusive thoughts in clinical disorders. Theory, Research and Treatment (pp.145-174). New York: Guilford Press.

Clark, D.A. y Purdon, C.L. (1995). The assessment of unwanted intrusive thoughts: A review and critique of the literature. Behaviour Research and Therapy, 33, 967-976.

Giménez, A., Morillo, C., Belloch, A., Lucero, M., Carrio, C. y Cabedo, E. (2003). Pensamientos intrusos en obsesivos subclínicos: contenidos, valoraciones, y estrategias de control. Revista De Psicopatología Y Psicología Clínica, 8, 23-38

Freeston, M.H., Ladouceur, R., Gagnon, F., y Thibodeau, N. (1991). Cognitive intrusions in a non-clinical population. I. Response style, subjective experience and appraisal. Behaviour Research and Therapy, 29, 585-597.

Freeston, M.H., Ladouceur, R., Thibodeau, N., y Gagnon, F. (1992). Cognitive iritrusions in a non-clirical population. Il. Associations with depressive, anxious, and compulsive symptom. Behaviour Research and Therapy, 30, 263-271.

Frost, R.O. y Hartl, T.L. (1996). A cognitive behavioral model of compulsive hoarding. Behaviour Research and Therapy, 34, 341-350.

Langlois, F., Freeston, M.H., y Ladouceur, R. (2000a). Differences and similarities between obsessive intrusive thoughts and worry in a non-clinical population: study 1. Behaviour Research and Therapy, 38, 157-173.

Langlois, F., Freeston, M.H., y Ladouceur, R. (2000b). Differences and similarities between obsessive intrusive thoughts and worry in a non-clinical population: study 2. Behaviour Research and Therapy, $38,175-189$.

Lucero,M. (2002). Pensamientos intrusivos en población general. Tesis Doctoral. Facultad de Psicología. Universitat de Valencia.

Morillo, C., Belloch, A. y Garcla-Soriano (2006). Clinical obsessions in obsessive-compulsive patients and obsession-relevant intrusive thoughts in non-clinical, depressed and anxious subjects: where are the differences?. Behaviour Research and Therapy. DOl:10.1016/j.brat.2006.11.005

Morillo, C., Giménez, A., Belloch, A., Lucero, M., Carrió, C., y Cabedo,

E. (2003). Pensamientos intrusos en obsesivos subclinicos: 
contenidos, valoraciones y estrategias de control. Revista de Psicopatología y Psicología Clínica, 8, 23-38.

Niler, F.R., y Beck, S.J. (1989). The relationship among guilt, dysphoria, anxiety and obsessions in a normal population. Behaviour Research and Therapy, 27, 213-220.

Parkinson, I., y Rachman, S. (1981). Part II. The nature of intrusive thoughts. Advances in Behaviour Research and Therapy, 3, 101-110

Purdon, C., y Clark, D. (1993). Obsessive intrusive thoughts in nonclinical subjects. Part I. Content and relation with depressive, anxious, and obsessional symptoms. Behaviour Research and Therapy, 31, 713-720.

Purdon, C., y Clark, D. (1994a). Obsessive Intrusive thoughts in nonclinical subjects. Part II. Cognitive appraisal, emotional response and thought control strategies. Behaviour Research and Therapy, $32,403-410$.

Purdon, C., y Clark, D. (1994b). Perceived control and appraisal of obsessional thoughts: A replication and extension. Behavioral and Cognitive Psychotherapy, 22, 269-285.

Purdon, C., y Clark, D.A. (1999). Metacognition and obsessions. Clinical Psychology and Psychotherapy, 6, 102-110.

Rachman, S. (1981). Part l-unwanted intrusive cognitions. Advances in Behaviour Research and Therapy, 3, 89-99.

Rachman, S. (1997). A cognitive theory of obsessions. Behaviour Research and Therapy, 35, 793-802.

Rachman, S. (1998). A cognitive theory of obsessions: elaborations. Behaviour Research and Therapy, 36, 385-401.

Rachman, S., y de Silva, P. (1978). Abnormal and normal obsessions. Behaviour Research and Therapy, 16, 233-248.

Rasmussen, S.A., y Eisen, J.L. (1990). Epidemiololgy of obsessive compulsive disorder. Journal of Clinicall Psychiatry, 51, 10-13

Reynolds, M., y Salkovskis, P.M. (1991). The relationship among guilt, dysphoria, anxiety and obsessions in a normal population: an attempted replication. Behaviour Research and Therapy, 30, 259265.

Salkovskis, P.M., y Harrison, J. (1984). Abnormal and normal obsessions-a replication. Behaviour Research and Therapy, 22, 549-552. 
Salkovskis, P.M. (1985). Obsessional-compulsive problems: A cognitive-behavioral analysis. Behaviour Research and Therapy, 23, 571-583.

Salkovskis, P.M. (1989). Cognitive-behavioral factors and the persistence of intrusive thoughts in obsessional problems. Behaviour Research and Therapy, 27, 677-682.

Scahill, L., Riddle, M., Hardin, M., Ort S.I., King, R.A., Goodman, W.K., et al (1997). Children's Yale Brown obsessive compulsive scale: Realiability and validity. J Am Acad Child Adolesc Psychiatry, 36, 844-52.

Toro, J.; Cervera, M.; Osejo, E., y Salamero, M. (1992). ObsessiveCompulsive Disorder in Childhood and Adolescence: a Clinical Study. Journal of Child Psychology and Psychiatry., 33, 10251037.

Turner, S.M., Beidel, D.C., y Stanley, M.A. (1992). Are obsessional thoughts and worry different cognitive phenomena?. Clinical Psychology Review, 12, 257-270.

Ulloa, R.E; de la Peña, F.; Higuera, F.; Palacios, L.; Nicolini, H., y Ávila, J.M. (2004). Estudio de validez y confiabilidad de la versión en español de la escala Yale-Brown del trastorno obsesivo-compulsivo para niños y adolescentes. Actas Españolas de Psiquiatría, 32, 216221. 